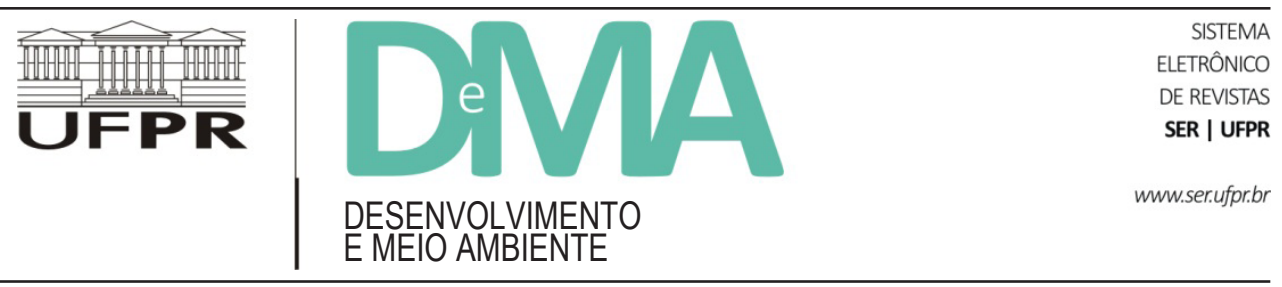

\title{
Pescadores da Praia Grande, Paraty, RJ: aspectos da resiliência em seu sistema socioecológico
}

\section{The Fishermen from Praia Grande, Paraty, RJ: Resilience Aspects in Their Socio-Ecological System}

\author{
Fernanda Pereira de Mesquita NORA ${ }^{1 *}$, Vinicius Figueiredo NORA², Mariana CLAUZET ${ }^{3}$, Milena RAMIRES ${ }^{2}$, \\ Alpina BEGOSSI ${ }^{2,4}$ \\ ${ }^{1}$ Programa de Pós-Graduação em Desenvolvimento Sustentável (PPG/CDS), Universidade de Brasília (UnB), Brasília, DF, Brasil. \\ ${ }^{2}$ Universidade Santa Cecília (UNISANTA), Santos, SP, Brasil. \\ ${ }^{3}$ Instituto de Ciência e Tecnologia em Políticas Públicas, Estratégias e Desenvolvimento (INCT/PPED), Universidade Federal do Rio de Janeiro \\ (UFRJ), Rio de Janeiro, RJ, Brasil. \\ ${ }^{4}$ Universidade Estadual de Campinas (UNICAMP), Campinas, SP, Brasil. \\ ${ }^{*}$ E-mail de contato: fernandamesquita84@gmail.com
}

Artigo recebido em 21 de junho de 2016, versão final aceita em 8 de março de 2017.

RESUMO: A resiliência, como propriedade de sistemas socioecológicos, está relacionada à capacidade que tais sistemas têm de se adaptar e lidar com perturbações. Este estudo examinou as características presentes em uma comunidade de pescadores artesanais por meio de alguns indicadores de resiliência (flexibilidade, capacidade de organização e capacidade de aprendizagem). O indicador flexibilidade representa a dependência econômica em relação ao recurso natural; o indicador capacidade de organização informa a respeito da participação em tomadas de decisão e em organizações comunitárias; e o indicador capacidade de aprendizagem refere-se à percepção das alterações ambientais em função da atividade humana. De forma conjunta, esses indicadores podem informar sobre a capacidade adaptativa da comunidade e sua relação com a gestão do recurso. Para esta investigação, entrevistas semiestruturadas foram realizadas nos meses de janeiro, abril, julho e agosto de 2012 na comunidade da Praia Grande e entorno (Paraty/RJ) $(n=22)$. Quanto ao indicador flexibilidade, 59,1\% dos pescadores entrevistados têm outra forma de obtenção de renda, sendo o turismo a principal delas (36,4\%). A maioria $(77,2 \%)$ utiliza mais de uma tecnologia de pesca, o que pode tanto favorecer a diminuição da pressão sobre o recurso pesqueiro como ter efeito contrário, se for o caso do uso de tecnologias mais intensivas, como $\mathrm{o}$ arrasto. Acerca da capacidade de organização dos pescadores, foi identificada e discutida a baixa participação em relação às tomadas de decisão em relação à pesca, em especial pelo fato de a área de estudo estar situada em um contexto de conflitos socioambientais com unidades de conservação. Quanto à capacidade de aprendizagem, os resultados indicam que os pescadores percebem a atividade humana como agente responsável por alterações no ambiente natural e sugerem diferentes soluções diante do decréscimo de cada espécie-alvo. Por meio do uso dos indicadores de resiliência, foi possível identificar os atributos que permitem a adaptação da 
comunidade diante de distúrbios e que têm o potencial de diminuir a pressão sobre os recursos pesqueiros, assim como aqueles que possuem efeito contrário em relação a esses recursos.

Palavras-chave: pesca artesanal; resiliência socioecológica; indicadores de resiliência; Paraty.

ABSTRACT: Resilience, as a property of social-ecological systems, is related to the ability of such systems to adapt and deal with perturbations. This study examined the characteristics in a community of artisanal fishermen, using some of the resilience indicators (flexibility, capacity to organize and capacity to learn). The flexibility indicator represents the economic dependence on the natural resource; the capacity to organize indicator informs about participation in decision-making and in community organizations; and the capacity to learn indicator refers to the perception of environmental alterations in relation to human activity. Together, these indicators can inform about the adaptive capacity of the community and its relationship with resource management. Semi structured interviews were conducted in the months of January, April, July and August of 2012 in the community of Praia Grande and surroundings (Paraty/RJ) (n=22). As to the flexibility indicator, 59.1\% of the fishermen interviewed have another source of income, being tourism the main activity among them (36.4\%). Most of the fishermen (77.2\%) use more than one type of fishing technique which may either reduce pressure on the fishing resource or have an opposite effect, if it is the case of the use of more intensive techniques, such as trawling. Regarding the fishermen's capacity to organize, it was found and discussed the low participation on decision-making regarding fishing, especially because the area of the study is situated in a context of social-environmental conflicts with conservation areas. As for the capacity to learn, the results indicate that the fishermen perceive the human activity as an agent responsible for the perturbations in the natural environment, and they suggest different solutions in face of the decrease of each target species. Through the use of the resilience indicators it was possible to identify the attributes that allow the adaptation of the community to perturbations and the ones that have the potential to reduce the pressure on the fishing resources, as well as those that have the opposite effect in relation to these resources.

Keywords: artisanal fishing; socio-ecological resilience; resilience indicators; Paraty.

\section{Introdução}

Estoques pesqueiros e comunidades pesqueiras, por estarem associados, representando sistemas socioecológicos (Begossi et al., 2012), devem fazer parte do manejo que objetiva a exploração sustentável dos recursos. Jentoft (2000, p. 54) enfatiza o papel fundamental das comunidades no manejo da pesca, ao assumir que "estoques pesqueiros viáveis necessitam de comunidades pesqueiras viáveis".

Sistemas socioecológicos (SSE) são aqueles nos quais sistemas ecológicos e sociais estão intrinsecamente ligados (Anderies et al., 2004). Um SSE é formado por um conjunto de subsistemas, que, ao interagirem, produzem resultados que afetam o sistema como um todo, cada subsistema, além de outros SSE menores ou maiores. Por definição, os subsistemas são: (a) sistemas de recursos (por exemplo, a pesca costeira), (b) unidades de recursos (por exemplo, as lagostas), (c) sistemas de governança (por exemplo, o governo e outras organizações responsáveis pela gestão da pesca) e, por fim, (d) os usuários (os pescadores). Cada subsistema é, ainda, formado por múltiplas variáveis, como, por exemplo, o tamanho do sistema de recursos, a mobilidade de uma determinada unidade de recurso e o conhecimento dos usuários (Ostrom, 2009).

Em relação a esses sistemas, o conceito de resiliência está relacionado à sua capacidade adaptativa, ou seja, à capacidade de aprendizado, de organização e adaptação frente a distúrbios, sem comprometimento de sua estrutura e função 
(Holling, 2001). Os objetivos de uma gestão com base na resiliência socioecológica seriam os de (a) impedir que o sistema mude para configurações indesejadas diante de perturbações externas e de (b) manter determinada configuração, que permite a reorganização do sistema após uma mudança maciça (Walker et al., 2002). Os distúrbios podem ser vistos, dentro desse enfoque, como oportunidades de mudança e transformação para estados mais desejados (Walker et al., 2002; Hughes et al., 2005).

A operacionalização do conceito por meio de indicadores é útil para a compreensão da complexidade e da diversidade de variáveis que podem afetar a resiliência de sistemas socioecológicos (Davidson et al., 2013). Alguns desses indicadores de resiliência foram desenvolvidos por diferentes autores, como o indicador flexibilidade (Gunderson, 1999), que diz respeito à variedade de formas de obtenção de renda; o indicador capacidade de organização (Carpenter et al., 2001), que se refere à capacidade de auto-organização, como as organizações comunitárias; o indicador capacidade de aprendizagem (Carpenter et al., 2001), relacionado à percepção de alterações ambientais; e o indicador patrimônio (Adger, 2000), que compreende aspectos de infraestrutura da comunidade. Em outras palavras, sistemas que apresentam diversificação econômica, capazes de se organizarem para tomada de decisões conjuntas e que percebem mudanças ambientais e propõem soluções diante dessas são mais resilientes, mais aptos à renovação diante de distúrbios.

No Brasil, há estudos sobre resiliência de comunidades costeiras por meio de diferentes abordagens: reconstrução histórica e uso do modelo de ciclos adaptativos na Reserva de Desenvolvimento Sustentável da Praia do Aventureiro, na Ilha Grande
(RJ) (Prado et al., 2015) e na Lagoa de Ibiraquera (SC) (Seixas \& Berkes, 2005); uso de indicadores de resiliência para comparar sistemas de comanejo pesqueiro em unidades de conservação de uso sustentável (Lopes et al., 2011) e análise da relação entre modos de vida e vulnerabilidade à insegurança alimentar em populações caiçaras $^{1}$ (Hanazaki et al., 2013).

O presente estudo examina, por meio de alguns indicadores de resiliência (flexibilidade, capacidade de organização e capacidade de aprendizagem), os atributos presentes em uma comunidade de pescadores artesanais de Paraty. Segundo as características de cada indicador, é possível compreender os fatores que colaboram para a adaptação frente a mudanças ou que, de maneira contrária, ameaçam a resiliência socioecológica do sistema.

\section{Material e métodos}

\section{1. Área de estudo: comunidade de pescadores da Praia Grande e entorno}

A comunidade de pescadores artesanais da

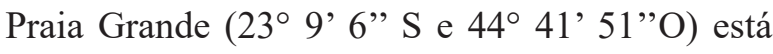
localizada na cidade de Paraty, litoral sul do Estado do Rio de Janeiro. A comunidade apresenta uma pequena estrutura comercial, contando com um mercado, uma padaria, uma peixaria, alguns bares e restaurantes. Na peixaria, transcorre a maioria dos desembarques pesqueiros da Praia Grande e da Ilha do Araújo. Esta última, adjacente à Praia Grande, encontra-se a cerca de cinco minutos de barco do continente (Lopes, 2010a). Praia Grande tem uma escola de ensino fundamental, um centro de infor-

\footnotetext{
${ }^{1}$ Os habitantes rurais nativos da costa sudeste da Mata Atlântica, descendentes de índios nativos (tupinambás) e portugueses (Begossi, 2006, p. 2).
} 
mática com acesso à Internet e um posto de saúde. Segundo Begossi (2010a), estima-se um número de 20 pescadores para a comunidade.

De acordo com Lopes (2010a), os pescados de maior importância para a comunidade da Praia Grande são camarão sete barbas, camarão-branco, corvina, pescada e tainha. A rede de espera e o arrasto são as principais tecnologias de captura. Para a comunidade da Ilha do Araújo, os pescados considerados mais importantes são robalo, camarão branco, corvina, cação e tainha.

Atualmente, as atividades de pesca desta e de outras comunidades de pescadores de Paraty são limitadas pela presença de uma unidade de conservação de proteção integral, a Estação Ecológica de Tamoios (ESEC de Tamoios, criada em 1990, mas efetivamente implementada a partir de 2008) (Lopes et al., 2013).

\subsection{Coleta de dados}

Os dados utilizados neste trabalho foram obtidos por meio de entrevistas semiestruturadas realizadas com pescadores que desembarcavam o pescado na Praia Grande, maiores de 18 anos; que pescavam em tempo integral ou parcial; praticavam a pesca há, no mínimo, 10 anos, e utilizavam apetrechos de pesca de pequena escala; pescavam utilizando barcos de pequeno porte na "costeira" ou em canoas a motor ou a remos; e não eram pescadores embarcados em traineiras e arrastos de grande porte (Begossi, 2010a). Para a seleção dos pescadores entrevistados, este estudo utilizou a técnica de amostragem snowball sampling (Biernacki \& Waldorf, 1981), conhecida como "método bola-de-neve". Para tanto, houve o contato inicial com um pescador artesanal indicado anteriormente por dois dos autores deste artigo, que realizavam pesquisa na comunidade desde 2009. Após a entrevista, solicitava-se ao pescador que indicasse outros pescadores artesanais, de acordo com a proposta da pesquisa. $\mathrm{O}$ pescador informava também onde o pescador indicado poderia ser encontrado, de modo que foram feitas entrevistas em diferentes locais (casa de pescador, píer, próximo à peixaria da Praia Grande, entre outros).

Os indicadores de resiliência utilizados seguiram a proposta de Cinner et al. (2009): flexibilidade, capacidade de organização e capacidade de aprendizagem. Esses indicadores foram escolhidos em função do que informam acerca da capacidade de adaptação do sistema e, ainda, em razão da praticidade em relação à coleta e análise dos dados. $\mathrm{O}$ indicador flexibilidade incluiu questões sobre o período de pesca e a diversidade de meios de subsistência. $\mathrm{O}$ indicador capacidade de organização envolveu dados a respeito da participação em organização comunitária, em processos de tomadas de decisão sobre a pesca e de organização para a conservação dos recursos pesqueiros. Por fim, a capacidade de aprendizagem foi investigada sob o aspecto da percepção pelos pescadores sobre os seres humanos como agentes transformadores do ambiente e, igualmente, sobre a abundância das espécies-alvo. Optou-se por não utilizar o indicador patrimônio. Segundo o método utilizado por Cinner et al. (2009), além da coleta de dados sobre a infraestrutura geral da comunidade, seriam necessárias, por meio de pesquisa domiciliar, informações a respeito dos bens e da estrutura das residências (material das paredes, do piso, do telhado). Como mencionado anteriormente, as entrevistas foram realizadas em diferentes locais, de acordo com a indicação dos pescadores. 
Em relação a cada indicador, as questões ${ }^{2}$ utilizadas nas entrevistas e analisadas no presente artigo foram:

(a) Flexibilidade: tempo dedicado à pesca (integral ou parcial); outras atividades; renda média mensal; tecnologias de pesca utilizadas.

b) Capacidade de organização: pescador que represente o grupo em reuniões; participação em alguma associação e/ou colônia e para que servem; "você acha que vale a pena pescar menos para preservar?"; "mesmo ganhando menos?"; "você acha que deve ser compensado por isso?".

c) Capacidade de aprendizagem: espécies-alvo da tecnologia de pesca utilizada; "a seu ver, tinha mais, menos, ou a mesma quantidade desta antigamente? ${ }^{3}$ Justificativa para a resposta anterior"; Para a resposta "diminuição da espécie", perguntar: "que medida você acha que poderia melhorar essa situação?”.

\subsection{Análise dos dados}

A análise dos dados usou a frequência de citações das respostas dadas, dispostas em tabelas e gráficos.

$\mathrm{Na}$ análise dos dados referentes ao indicador de resiliência flexibilidade, foi utilizado o teste $\mathrm{t}$ de Student (Zar, 1999), para verificar as diferenças entre os valores médios de renda financeira. O nível de significância considerado foi $95 \%$. O teste foi feito por meio do software Bioestat 5.3.

A identificação do pescado citado pelo pescador (nome popular) foi feita por meio dos resultados de identificação das espécies desembarcadas na Praia Grande (Begossi et al., 2014). Para os pescados não identificados no estudo anteriormente citado (lula e camarão), utilizou-se a lista presente em publicações anteriores (Lopes, 2010b, p. 175), cuja identificação foi baseada em trabalhos publicados.

\section{Resultados}

Foram entrevistados 22 pescadores - 13 pescadores da comunidade da Praia Grande e 9 do entorno ( 8 pescadores da Ilha do Araújo e 1 pescador da comunidade da Barra Grande). A idade média dos entrevistados foi de 58,5 anos $( \pm 12,3)$ (idade mínima de 40 anos e máxima de 89 anos). O tempo médio por eles dedicado à pesca foi de 44,4 anos $( \pm 12,9)$ (mínimo de 20 anos e máximo de 79 anos). Em relação à escolaridade, a maioria tem o ensino fundamental incompleto $(72,7 \%)$.

\subsection{Indicadores de resiliência: flexibilidade}

Dos pescadores entrevistados $(n=22), 59,1 \%$ pescam em tempo parcial, dedicando-se também a outras atividades, entre as quais o turismo representou a maior parcela $(36,4 \%)$, seguido pela categoria outros $(13,6 \%)$, que engloba atividades

\footnotetext{
2 Parte da pesquisa de dissertação de mestrado de um dos autores deste artigo.

${ }^{3}$ Nota-se que, na pergunta, não foi estabelecido um marco temporal específico em relação ao termo "antigamente", e isso tem relação com o fato de os pescadores, frequentemente, marcarem o tempo pelos eventos mais significativos que ocorreram ao longo de seus cursos de vida e não pela exatidão dos anos ou décadas (Filgueiras, 2007). Assim, buscou-se que o próprio pescador dissertasse livremente sobre as alterações observadas em relação à "antigamente". Na pesquisa de mestrado que deu origem ao presente artigo, observou-se que apenas dois pescadores mencionaram décadas específicas para descrever tecnologias de pesca não utilizadas atualmente.
} 
com uma citação cada (trabalho em bar; pedreiro e jardinagem) e carpintaria $(9,1 \%)$.

A renda média mensal dos pescadores que se dedicam integralmente à pesca foi de $\mathrm{R} \$ 910,66$ ( \pm $255,85),{ }^{4}$ enquanto a daqueles que complementam a pesca com outras atividades foi de $\mathrm{R} \$ 1.128,00$ $( \pm 397,10)$, não havendo diferença significativa entre os dois grupos $(\mathrm{p}=0,244 ; \mathrm{t}=1,21 ; \mathrm{gl}=16)$.

A maioria dos pescadores entrevistados utiliza três ou mais tecnologias de pesca $(54,5 \%)$, seguindo-se por aqueles que utilizam duas $(22,7 \%)$ e uma tecnologia $(22,7 \%)$.

As rendas citadas foram divididas em duas categorias: renda média inferior à $\mathrm{R} \$ 1.000,00 \mathrm{e}$ renda média superior ou igual à $\mathrm{R} \$ 1.000,00$. Assim, estabeleceu-se uma comparação dessas categorias com a frequência das atividades citadas pelo pescador (o que incluiu também o número de tecnologias de pesca). Dos pescadores entrevistados, quatro não souberam estimar ou não responderam a sua renda mensal (Tabela 1).

\subsection{Indicadores de resiliência: capacidade de organização}

No que se refere aos resultados sobre a representatividade dos pescadores em relação às decisões sobre a pesca, a maior parte dos entrevistados $(n=19)(86,4 \%)$ disse não haver na comunidade representante em reuniões. Daqueles que responderam haver um representante $(n=2)(9,1 \%)$, um mencionou o presidente da colônia dos pescadores e o outro citou um pescador da Ilha do Araújo. Apenas um pescador respondeu não saber se havia alguém que os representasse em reuniões referentes a decisões sobre a pesca.
TABELA 1 - Frequência das atividades citadas pelos pescadores, dividid3os em dois grupos: aqueles com renda média mensal inferior a $\mathrm{R} \$ 1.000,00 \mathrm{e}$ aqueles com renda média mensal superior ou igual a R\$1.000,00. São informados a média da renda e o desvio padrão por grupo $(\mathrm{n}=18)$.

\begin{tabular}{lcc}
\hline \multicolumn{1}{c}{ Atividades } & $\begin{array}{c}\text { Renda }<\text { R\$ 1.000,00 - } \\
\mathrm{R} \$ 701,57( \pm 137,03)\end{array}$ & $\begin{array}{c}\text { Renda } \geq \mathbf{R S} \\
\mathbf{1 . 0 0 0 , 0 0}-\mathrm{R} \$ 1.280,00 \\
( \pm 264,97)\end{array}$ \\
\hline Rede de espera & $29,60 \%$ & $28,60 \%$ \\
Arrasto & $7,40 \%$ & $16,70 \%$ \\
Linha & $7,40 \%$ & $11,90 \%$ \\
Espinhel & $7,40 \%$ & $7,10 \%$ \\
Zangarelho & - & $9,50 \%$ \\
Cerco & $7,40 \%$ & - \\
Tarrafa & - & $4,80 \%$ \\
Turismo & $7,40 \%$ & $11,90 \%$ \\
Aposentadoria & $14,80 \%$ & - \\
Outros (n=1 cada) & $18,50 \%$ & $9,50 \%$ \\
Total de \\
$\begin{array}{l}\text { atividades } \\
\text { (citações) }\end{array}$
\end{tabular}

Perguntados se participavam de alguma associação e/ou da colônia de pescadores, a maioria disse fazer parte da colônia $(n=15)(68,2 \%)$. Além da colônia, um pescador informou fazer parte do Conselho da ESEC de Tamoios e que participa de reuniões do IBAMA relacionadas à temática da pesca. A maior parte dos pescadores $(41,7 \%)$ justificou a participação na colônia em função da aposentadoria, seguido por "orientação a documentação" (12,5\%). Apenas dois pescadores (8,3\%) disseram participar, por meio da colônia, de palestras e reuniões referentes à troca de opiniões e decisões sobre a pesca.

\footnotetext{
${ }^{4}$ Salário mínimo vigente em 2012: R\$ 622,00 (Decreto No 7.655 de 23.12.2011).
} 
Os entrevistados mostraram disposição para pescar menos em benefício da conservação dos estoques pesqueiros locais. Para 59,1 \% dos entrevistados $(n=13)$, vale a pena pescar menos para preservar, mas para $13,6 \%(n=3)$, não. O restante, equivalendo a $27,3 \%(n=6)$, apresentou justificativas que não podiam ser enquadradas nas categorias anteriores, por apresentarem condicionantes em suas respostas, dispostas na Tabela 2.

TABELA 2 - Frequência dos motivos apresentados pelos pescadores $(\mathrm{n}=22)$ em resposta à pergunta: "Você acha que vale a pena pescar menos para preservar?". Os números entre parênteses referem-se ao número de pescadores.

\begin{tabular}{|c|c|c|}
\hline $\operatorname{sim}(n=13)$ & $\%$ & NC* \\
\hline não detalharam & $42,9 \%$ & 6 \\
\hline aumento do preço do camarão & $21,4 \%$ & 3 \\
\hline necessidade de conscientização & $7,1 \%$ & 1 \\
\hline demarcar área de pesca & $7,1 \%$ & 1 \\
\hline manutenção da pesca a longo prazo & $7,1 \%$ & 1 \\
\hline em relação ao arrasto e cerco & $7,1 \%$ & 1 \\
\hline aumentar o período de defeso do camarão & $7,1 \%$ & 1 \\
\hline Total & & 14 \\
\hline \multicolumn{3}{|l|}{ não $(n=3)$} \\
\hline escassez do recurso & $33,3 \%$ & 1 \\
\hline proibição somente no defeso & $33,3 \%$ & 1 \\
\hline condições de vida do pescador & $33,3 \%$ & 1 \\
\hline Total & & 3 \\
\hline \multicolumn{3}{|l|}{ depende $(n=6)$} \\
\hline depende da espécie & $33,3 \%$ & 2 \\
\hline não detalharam & $33,3 \%$ & 2 \\
\hline depende do sistema de captura & $16,7 \%$ & 1 \\
\hline depende do tamanho & $16,7 \%$ & 1 \\
\hline Total & & 6 \\
\hline
\end{tabular}

FONTE: Os autores.

$\mathrm{NC}^{*}$ : número de citações
Em relação a uma possível redução da renda mensal decorrente de uma pesca menos intensa, os resultados mostram que houve um crescimento do número daqueles que, diante dessa nova condição, consideraram que não valeria a pena pescar menos para preservar $(n=6)(27,3 \%)$. Entretanto, a maioria dos pescadores $(n=13)(59,1 \%)$ manteve a sua posição, mesmo com a perspectiva de se ganhar menos. Três pescadores $(n=3)$ não deixaram clara a sua resposta, de modo que foram enquadrados na categoria "outros". A frequência dos motivos levantados para cada categoria de resposta (sim e não) está disposta na Tabela 3 .

TABELA 3 - Frequência dos motivos apresentados pelos pescadores para a pergunta: "Você acha que vale a pena pescar menos para preservar? Mesmo ganhando menos?" (n=22). Os números entre parênteses referem-se ao número de pescadores.

\begin{tabular}{lcc}
\hline sim $\mathbf{( n = 1 3 )}$ & $\mathbf{\%}$ & $\mathbf{N C}$ \\
\hline não detalharam & $69,2 \%$ & 9 \\
aumento do preço de mercado & $15,4 \%$ & 2 \\
a renda tem de ser complementada & $7,7 \%$ & 1 \\
necessidade de conscientização & $7,7 \%$ & 1 \\
Total & & $\mathbf{1 3}$ \\
\hline não $(\mathbf{n}=\mathbf{6})$ & & \\
não detalharam & $67,0 \%$ & 4 \\
só se for compensado & $17,0 \%$ & 1 \\
"e aí vai viver como?" & $17,0 \%$ & 1 \\
Total & & $\mathbf{6}$ \\
\hline outros $(\mathbf{n}=\mathbf{3})$ & & \\
não deixou claro & $100,0 \%$ & 3 \\
Total & & $\mathbf{3}$ \\
\hline
\end{tabular}

FONTE: Os autores.

Em contrapartida, os resultados mostram que a maioria dos pescadores $(n=19)(86,4 \%)$ considera que deve ser compensada por pescar menos. As justificativas variaram, mas a maior frequência está 
relacionada à conservação dos peixes $(31,8 \%)$. Os pescadores que não acham que devem ser compensados $(n=3)(13,6 \%)$ apresentaram diferentes justificativas ( $\mathrm{n}=1$ cada), as quais estão discriminadas na Tabela 4.

TABELA 4 - Motivos apresentados pelos pescadores $(n=22)$ em resposta à pergunta: "Você acha que deve ser compensado por isso? Por quê?" Os números entre parênteses referem-se ao número de pescadores.

\begin{tabular}{|c|c|c|}
\hline $\operatorname{sim}(19)$ & $\%$ & NC \\
\hline para a conservação do recurso & $31,8 \%$ & 7 \\
\hline $\begin{array}{l}\text { verba para comprar outro material } \\
\text { (pescadores de arrasto) }\end{array}$ & $9,1 \%$ & 2 \\
\hline melhorar a pesca & $9,1 \%$ & 2 \\
\hline para complementar a renda & $9,1 \%$ & 2 \\
\hline para compensar a perda de dinheiro & $4,5 \%$ & 1 \\
\hline $\begin{array}{l}\text { para todos os peixes, como no caso do } \\
\text { camarão (defeso) }\end{array}$ & $4,5 \%$ & 1 \\
\hline $\begin{array}{l}\text { "receber até o camarão crescer e pegar de } \\
\text { redinha" }\end{array}$ & $4,5 \%$ & 1 \\
\hline aumentar o defeso & $4,5 \%$ & 1 \\
\hline ajuda do governo & $4,5 \%$ & 1 \\
\hline "o governo tem dinheiro" & $4,5 \%$ & 1 \\
\hline pescando menos, o preço aumenta & $4,5 \%$ & 1 \\
\hline "quanto mais compensado melhor" & $4,5 \%$ & 1 \\
\hline manter a pesca a longo prazo & $4,5 \%$ & 1 \\
\hline Total & & 22 \\
\hline \multicolumn{3}{|l|}{ não (3) } \\
\hline $\begin{array}{l}\text { o próprio pescador deveria compensar com } \\
\text { outra atividade }\end{array}$ & $33,3 \%$ & 1 \\
\hline deve-se viver daquilo que produz & $33,3 \%$ & 1 \\
\hline $\begin{array}{l}\text { tem que ter consciência (não matar peixe } \\
\text { pequeno) }\end{array}$ & $33,3 \%$ & 1 \\
\hline Total & & 3 \\
\hline
\end{tabular}

FONTE: Os autores.

\subsection{Indicador de resiliência: capacidade de aprendizagem}

Sobre a abundância das espécies-alvo citadas pelos pescadores, os entrevistados foram indagados a respeito da abundância atual dessas espécies em relação à de antigamente. Cabe destacar que cada pescador opinou somente a respeito das espécies-alvo das tecnologias de pesca que eles mesmos praticam. Nenhum pescador relatou maior abundância da espécie-alvo (Tabela 5). Dos pescados citados $(\mathrm{n} \geq 2)$, apenas a lula (Loligo spp.) foi percebida pela maioria dos pescadores $(75 \%)$ como tendo abundância atual igual à de antigamente. Dos pescadores de garoupa (Epinephelus marginatus), apenas um pescador considera que a abundância desse pescado permanece a mesma do passado. Este foi também o pescador com menor tempo dedicado à pesca entre os entrevistados (20 anos). Os resultados em relação aos demais pescados apontam que os pescadores percebem uma redução de sua abundância.

As justificativas para a redução dos estoques da população variaram de acordo com a espécie-alvo (Tabela 6), e as maiores frequências de citação estão relacionadas a tecnologias de pesca específicas (arrasto, parelha, traineira, mergulho, cerco com mergulho).

Aos pescadores que apontaram o decréscimo de espécies-alvo das tecnologias por eles praticadas, foi perguntado que solução poderia ser aplicada para melhorar a situação. Os resultados indicam variação nas soluções de acordo com o pescado (Tabela 7). 
TABELA 5 - Percepção da abundância atual da espécie-alvo em relação ao passado. Foram considerados apenas os pescados com duas ou mais citações referente à pergunta "A seu ver, tinha mais, menos ou a mesma quantidade desta espécie antigamente?" (n=22)

\begin{tabular}{|c|c|c|c|c|}
\hline \multirow[t]{2}{*}{ Pescado ( $n \geq 2$ citações) } & \multirow[t]{2}{*}{ Nome científico } & \multicolumn{3}{|c|}{$\begin{array}{c}\text { Tinha mais, menos ou a mesma } \\
\text { quantidade? }\end{array}$} \\
\hline & & Mais & Igual & $\mathrm{NC}$ \\
\hline Corvina & Micropogonias furnieri; Ophioscion punctatissimos & $100 \%$ & & 18 \\
\hline Camarão-branco & Litopenaeus schmitti & $100 \%$ & & 15 \\
\hline Camarão-sete-barbas & Xiphopenaeus kroyeri & $100 \%$ & & 11 \\
\hline Vermelho & Lutjanus spp. & $100 \%$ & & 10 \\
\hline Garoupa & Epinephelus marginatus & $80 \%$ & $20 \%$ & 4 \\
\hline Prejereba & Lobotes surinamensis & $100 \%$ & & 4 \\
\hline Bagre & Sciadeichtrhys luniscutis; Aspistor luniscutis; Sciades passany & $100 \%$ & & 3 \\
\hline Total & & & & 120 \\
\hline
\end{tabular}

FONTE: Os autores.

TABELA 6 - Principais justificativas apontadas pelos pescadores para a redução das espécies-alvo das tecnologias de pesca empregadas. Os pescados apresentados são aqueles que foram citados por dois ou mais pescadores.

\begin{tabular}{|c|c|c|c|c|}
\hline \multirow{2}{*}{ Pescados } & \multicolumn{3}{|c|}{ Principais justificativas para o decréscimo da população-alvo } & \multirow{2}{*}{ NC } \\
\hline & Justificativa 1 & Justificativa 2 & Justificativa 3 & \\
\hline Corvina & Traineira $(31 \%)$ & Pesca industrial $(19 \%)$ & Arrasto $(8 \%)$ & 48 \\
\hline Camarão-branco & $\begin{array}{l}\text { Arrasto }(32 \%) \text { e maior esforço de pesca } \\
\qquad(32 \%)\end{array}$ & $\begin{array}{l}\text { Não cumprimento do defeso } \\
(8 \%) \text {, pesca predatória }(8 \%) \text { e } \\
\text { traineira }(8 \%)\end{array}$ & Outras ( 1 citação cada) & 25 \\
\hline Robalo-peba & Cerco c/ mergulho (57 \%) & Pesca predatória $(17 \%)$ & Arrasto $(9 \%)$ & 23 \\
\hline $\begin{array}{l}\text { Camarão-sete- } \\
\text { barbas }\end{array}$ & Maior esforço de pesca (39\%) & Arrasto $(28 \%)$ & $\begin{array}{c}\text { Não cumprimento do defeso }(11 \%) \\
\text { Pesca predatória }(11 \%)\end{array}$ & 18 \\
\hline Vermelho & Traineira $(40 \%)$ & Parelha $(27 \%)$ & Pesca predatória $(20 \%)$ & 15 \\
\hline Cação & Pesca industrial $(78 \%)$ & Maior esforço de pesca $(14 \%)$ & Maior esforço de pesca (14\%) & 14 \\
\hline Bagre & Não sei $(60 \%)$ & Arrasto $(40 \%)$ & & 5 \\
\hline Badejo & Pesca com mergulho $(50 \%)$ & Outras (uma citação cada) & & 4 \\
\hline Total & & & & 199 \\
\hline
\end{tabular}

FONTE: Os autores. 
TABELA 7 - Soluções apontadas pelos pescadores para a redução da espécie-alvo das tecnologias empregadas. Os pescados apresentados são aqueles que foram citados por dois ou mais pescadores e para os quais foram apresentadas soluções. Em destaque, estão as soluções com maiores frequências para cada pescado.

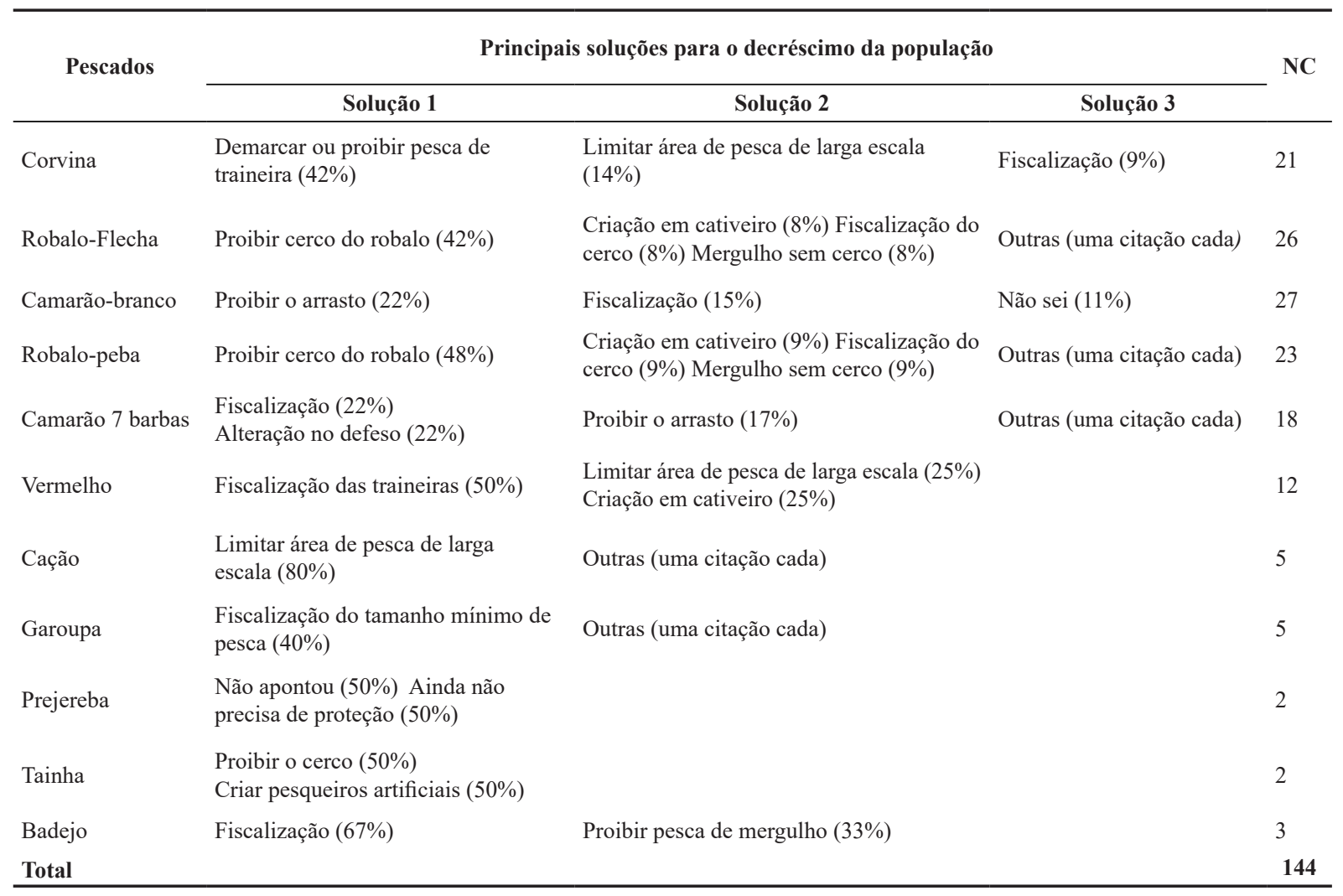

FONTE: Os autores.

\section{Discussão}

\subsection{Indicadores de resiliência: Flexibilidade}

A presença do turismo como principal atividade complementar desempenhada pelos pescadores da comunidade da Praia Grande confirma resultados anteriores para a comunidade estudada (Lopes, 2010a) e reflete a importância dessa atividade no cenário de Paraty como um todo (Breton \& Plante, 2005). Ou seja, diante da incerteza da produção pesqueira, a comunidade desenvolve outras formas de obtenção de renda, a fim de diminuir o risco associado à dependência exclusiva de uma única atividade (Bailey \& Pomeroy, 1996; Adger, 2000; Lopes, 2010a). Trimble \& Johnson (2013) mostraram que pescadores de Paraty estão se movendo para diferentes ocupações, embora demonstrem querer continuar a pescar no futuro. Entretanto, o mesmo estudo mostrou que os pescadores não consideram a pesca uma opção viável para seus filhos.

Outro ponto que merece destaque é a relação entre o ambiente em que os pescadores se encontram 
(ecossistema costeiro) e a possibilidade de diversificação econômica. Nesse sentido, Bailey \& Pomeroy (1996) destacaram o aspecto resiliente de comunidades costeiras do sudeste da Ásia em função de se localizarem em um ambiente altamente complexo e produtivo, que permitia a exploração de vários nichos econômicos, proporcionando, assim, uma menor vulnerabilidade do sistema social. Begossi (2006) destaca que a alta resiliência dos Caiçaras se relaciona à sua flexibilidade e à sua adaptação aos ciclos econômicos, além de outros fatores. Lopes et al (2011), ao utilizarem o conceito de resiliência socioecológica para compararem os sistemas de comanejo de duas categorias de unidades de conservação de uso sustentável no Brasil (Reservas Extrativista e de Desenvolvimento Sustentável), concluíram que, nas reservas amazônicas, as pessoas exploram uma grande diversidade de recursos naturais, se comparadas às da costa, as quais concentram-se mais na pesca. Entretanto, os autores ressaltaram que as pessoas que vivem em reservas costeiras podem se aproveitar do fato de estarem próximas aos centros urbanos para explorarem atividades relacionadas ao turismo, incluindo o ecoturismo. É preciso notar, no entanto, que, a despeito da riqueza do ambiente costeiro, na comunidade da Praia Grande, ainda há uma parcela de pescadores (40,9\%) dependentes exclusivamente da pesca, o que sugere novas questões para investigação. $\mathrm{O}$ declínio de importantes espécies-alvo percebido pelos pescadores (ver indicador capacidade de aprendizagem) expõe a vulnerabilidade do sistema ecológico e, consequentemente, do sistema social dependente desses recursos.

Ainda sob o enfoque da dependência econômica, destaca-se o aspecto positivo de se utilizar diferentes tecnologias de pesca, como ocorre na comunidade da Praia Grande, a fim de reduzir o risco associado à incerteza da exploração de um único recurso ou espécie e minimizar a pressão sobre um determinado recurso pesqueiro. Assim, do ponto de vista ecológico, espécies generalistas ou usuários de recursos que visam mais de uma espécie podem exibir um caráter mais resiliente, uma vez que podem alterar seus alvos de captura quando a necessidade surgir (Marshall et al., 2007).

A participação relativa de cada atividade exercida variou entre as duas categorias (pescadores com renda menor que $\mathrm{R} \$ 1.000,00$ e pescadores com renda maior ou igual a $\mathrm{R} \$ 1.000,00)$. O primeiro grupo está predominantemente concentrado em uma só tecnologia de pesca (rede de espera) e em uma renda fixa (aposentadoria). Para os pescadores que apresentaram renda média superior à $\mathrm{R} \$ 1.000,00$, rede de espera, arrasto, turismo e linha são mais relevantes. Observa-se que, diante da certeza de uma renda fixa (a aposentadoria), o pescador aposentado se concentra mais em uma determinada atividade. Em caso contrário, apresenta uma maior diversidade de formas de obtenção de renda. Além de diversificar as atividades, esse pescador utiliza tecnologias mais intensivas, como o arrasto. Por exemplo, McCay (1978), ao observar as respostas frente ao declínio na abundância de peixes em Newfoundland (Canadá), identificou duas estratégias gerais: a da diversificação (expressa por meio do pluralismo ocupacional, da implantação de diferentes tecnologias de pesca) e a da intensificação (na qual se investe mais em uma atividade). A autora identificou que a primeira estratégia a ser adotada era a da diversificação, em função do maior custo envolvido na intensificação (como, por exemplo, o investimento em barcos maiores). Ela concluiu que a estratégia da intensificação tende a piorar a tendência do declínio das capturas, por promover o aumento do esforço de pesca.

Não houve diferença entre as rendas médias mensais daqueles que pescam integralmente e dos 
que realizam outras atividades, o que sugere que a diversificação de atividades não resulta em incremento significativo na economia mensal. Entretanto, as estratégias individuais de diversificar (usar várias tecnologias) e intensificar (usar o arrasto) afetariam a resiliência do sistema socioecológico de duas formas opostas. A diversificação minimizaria o impacto sobre os recursos e a intensificação aumentaria esse impacto. No presente trabalho, a pesca de arrasto foi apresentada como justificativa para o declínio de 6 dos 12 pescados citados pelos pescadores (Tabela 6).

Adicionalmente, é importante ressaltar que, uma vez que o turismo representa um setor econômico importante para a comunidade e tem, nesse caso, o potencial de reduzir a dependência em relação à pesca, é necessário compreender de que forma essa atividade vem sendo desenvolvida na região. Em pesquisa anterior na comunidade da Praia Grande, Lopes (2010a) relatou que a prática do turismo se dava essencialmente por meio da locação de barcos para passeios e pesca esportiva. Diegues (1983) salienta que o domínio da arte da atividade pesqueira exige dos pescadores qualidades físicas e intelectuais aprimoradas para seu exercício. Assim, assumir tais pontos poderia ser interessante no desenvolvimento do turismo de base comunitária (TBC), por exemplo, no qual o patrimônio comunitário $^{5}$ representa a matéria-prima fundamental. No Brasil, essa forma de turismo tem recebido destaque por permitir "um desenvolvimento social mais justo e ambientalmente responsável", ao contrário do turismo massificado (Burgos \& Mertens, 2015). Isso faz com que a atividade turística ganhe uma dimensão que vai além das práticas de passeios de barco (característica do turismo convencional em comunidades costeiras) e possibilite a conservação de modos de vida e da biodiversidade, maximizando-se as formas de uso de um ambiente naturalmente diverso, como o existente na região. Dentro desse contexto, é importante salientar que o desenvolvimento desordenado do turismo de pesca, por exemplo, poderia levar a outros tipos de conflitos, como a disputa por pontos de pesca. Adicionalmente, seria importante utilizar mecanismos de compensação ambiental, que diversificariam as atividades e seriam auxiliares ao ordenamento ou manejo da pesca na região (Begossi et al., 2011).

No contexto do desenvolvimento turístico na região, outro ponto que merece atenção é o da infraestrutura básica. Nesse sentido, Lopes (2010a) relata que a comunidade da Praia Grande conta com coleta de lixo e abastecimento de água, mas a coleta de esgoto não é acessível a todas as casas (apenas $36 \%$ dos pescadores entrevistados a tinham). Assim, uma vez que as condições sanitárias influenciam não somente o turismo, mas também a qualidade dos recursos extraídos desse ecossistema, investimentos na infraestrutura desses locais são fundamentais para que as comunidades continuem a desenvolver formas de renda complementares. Adger (2000) ressalta que, ainda que as comunidades costeiras sejam mais resilientes, a sua economia ainda se baseia em um único sistema costeiro, de tal forma que um prejuízo ambiental afeta não somente as praias turísticas como também os recursos pesqueiros, além de causar outros impactos ecológicos.

\footnotetext{
5 “O patrimônio comunitário é formado por um conjunto de valores e crenças, conhecimentos e práticas, técnicas e habilidades, instrumentos e artefatos, lugares e representações, terras e territórios, assim como todos os tipos de manifestações tangíveis e intangíveis existentes em um povo. Através disso, se expressam seu modo de vida e organização social, sua identidade cultural e suas relações com a natureza" (Maldonato, 2009, p. 29).
} 


\subsection{Indicadores de resiliência: capacidade de organização}

Apesar de a maioria dos pescadores $(68,2 \%)$ afirmar que participa da colônia, os principais motivos levantados para essa participação referiram-se à aposentadoria $(41,7 \%)$ e à orientação sobre documentação (12,5\%). Apenas dois pescadores incluíram em suas justificativas a participação em palestras e reuniões; e, desses últimos, apenas um fez referência específica à troca de informações e decisões sobre a pesca (este pescador, além da colônia, participa do conselho da ESEC de Tamoios). Ressalte-se, ainda, que a maioria dos entrevistados disse não ter representantes em reuniões.

Trimble (2013) pesquisou as barreiras e oportunidades para a cogestão adaptativa da pesca em Paraty (Praia Grande e Ilha do Araújo) e Piriápolis (Uruguai). Os resultados indicaram que os pescadores estudados estão fracamente organizados e que as conexões em nível local foram prejudicadas pelo impacto negativo de agentes externos (relações conflituosas entre pescadores e agências governamentais relacionadas à pesca). No estudo da autora, os pescadores dessas comunidades também levantaram razões para não participarem de reuniões sobre a pesca, entre as quais se destacam: a ausência de associação/organização dos pescadores; a percepção de que pescadores de pequena escala não são ouvidos; de que somente a opinião do governo é levada em consideração; de que as soluções não são encontradas; de que as reuniões são apenas informativas ou consultivas, pois as decisões já foram tomadas; entre outras. Essas razões, levantadas pelos próprios pescadores, indicam a sua baixa confiança em relação aos órgãos governamentais que regulamentam a pesca e como essa fragilidade da relação e a falta de diálogo implicam baixa participação ou uma participação não direta nos processos e nas tomadas de decisão. De acordo com Adger et al (2005), processos caracterizados por assimetria de poder tendem a minar a confiança em mecanismos de gestão de recursos. No presente estudo, constatamos que essa assimetria pode limitar a capacidade adaptativa da comunidade diante de mudanças de cenário.

Outros estudos indicaram uma relação conflituosa entre os pescadores da região e as unidades de conservação (Oliveira, 2010; Lopes et al., 2013; Joventino et al., 2013), principalmente em função da não inclusão dos pescadores no processo de criação dessas áreas. Trimble (2013) também relatou que alguns pescadores da Praia Grande e da Ilha do Araújo explicaram que a participação do pescador se dá por meio da colônia e que é esta que interage com o Governo. No presente estudo, apenas dois pescadores incluíram a participação em palestras e reuniões sobre decisões relacionadas à pesca como justificativa para fazerem parte da colônia.

Em relação à organização para a conservação dos recursos pesqueiros, os resultados indicam que a maior parte dos pescadores $(59,1 \%)$ considera valer a pena pescar menos com o objetivo de conservar os recursos pesqueiros. Uma parcela de $27,3 \%$ dos pescadores condicionou a redução da pesca a algumas variáveis, como o tamanho dos peixes, a espécie e o sistema de captura. Isso sedimenta o fato de que os próprios pescadores, com base no conhecimento das espécies-alvo, podem apontar mecanismos eficientes de manejo pesqueiro (Begossi, 1995; Johannes et al., 2000; Drew, 2005; Silvano et al., 2006; Begossi, 2010b). Entretanto, diante da condição de se ganhar menos, o número de pescadores que consideraram não valer a pena a redução aumentou em relação à pergunta anterior.

A esse respeito, Ostrom (2000) destaca que, em um grupo, há indivíduos mais dispostos do que outros a iniciarem a reciprocidade a fim de se alcançar os benefícios da ação coletiva. Berkes et 
al (2006) argumentam que, no uso de um recurso comum, o maior problema enfrentado pelos indivíduos de um grupo é como se organizar para mudar de uma situação de ação individual para uma de ação coletiva. Segundo os autores, uma estratégia de ação coletiva é aquela que promove mais benefícios conjuntos e reduz os custos conjuntos (ou seja, a depleção do recurso). Enfatizam, ainda, que usuários organizados em uma comunidade aumentam as chances de sucesso na organização que objetiva a ação coletiva, por compartilharem estilos de vida e valores semelhantes, o que facilitaria a fiscalização das sanções e a solução de conflitos.

Perguntados se o pescador deveria ser compensado por pescar menos, a maioria foi favorável. A conservação dos recursos pesqueiros foi a principal justificativa mencionada, seguida, em menor frequência, pela melhoria da pesca e pela complementação de renda. Dois pescadores de arrasto mencionaram, ainda, que a compensação poderia vir na forma de recurso financeiro para a compra de outro material de pesca, como uma rede de espera. Esse resultado vai ao encontro de uma das propostas levantadas por Jennings \& Revill (2007) em relação aos incentivos necessários para a utilização de tecnologias de menor impacto: nesse caso, trata-se da concessão de subsídios para que os pescadores obtenham uma tecnologia mais sustentável. Os resultados supracitados parecem sugerir que os pescadores apresentam uma boa percepção a respeito da funcionalidade de mecanismos de compensação por serviços ambientais. Estudos recentes têm destacado medidas de comanejo pesqueiro envolvendo uma associação de instrumentos (policy mix), como os pagamentos por serviços ambientais (PSA) e acordos de pesca (AP), como uma forma de se conciliar conservação da biodiversidade associada à manutenção da pesca artesanal. Nesse sentido, ocorre a compensação por perdas associadas às restrições no acesso ao recurso (PSA) em troca de gestão comunitária e monitoramento de estoques pesqueiros (AP) (Begossi et al., 2011).

\subsection{Capacidade de aprendizagem-} percepção dos seres humanos como agentes transformadores do ambiente e da abundância das espécies-alvo

Sobre a abundância das espécies-alvo em relação a tempos passados, nenhum pescador relatou crescimento. Apenas a lula (Loligo spp.) foi percebida pela maioria dos pescadores de lula como não tendo alterado sua abundância em relação ao passado. Em estudo anterior na Baía da Ilha Grande, a lula esteve entre os poucos pescados citados entre aqueles que aumentaram em quantidade (Oliveira, 2010). Entretanto, o autor levanta a hipótese de essa percepção ter sido influenciada pelo fato de as entrevistas terem ocorrido à época de pesca da lula. Ainda, segundo o autor, a maioria dos pescadores artesanais entrevistados em Paraty respondeu que todos os peixes diminuíram em quantidade. Em seguida, foram citados a corvina, o camarão e o robalo. De acordo com o autor, para a comunidade da Praia Grande e Ilha do Araújo, o principal pescado citado em relação ao decréscimo foi o camarão.

Em relação às razões apontadas pelos pescadores para o decréscimo das espécies-alvo, em que pese a variação observada em função da espécie em questão, de maneira geral, pode-se afirmar que estiveram relacionadas a tecnologias específicas. Merece destaque o fato de tecnologias seletivas, como o mergulho e o cerco com mergulho, terem sido consideradas como as principais responsáveis pela diminuição de pescados comercialmente importantes, como garoupas e badejos (em relação ao mergulho) e robalos (cerco com mergulho - técnica 
especialmente utilizada por pescadores da comunidade de Tarituba, ao norte de Paraty, considerados por outros pescadores como especialistas em relação a esta tecnologia [Lopes, 2010b, Nora, 2013]). Isso enfatiza a questão de se avaliar o método empregado na captura e a biologia da espécie-alvo em questão. Assim, garoupas e badejos, embora capturados a partir de tecnologias seletivas, são extremamente vulneráveis à sobrepesca, principalmente devido à maturidade sexual tardia, à longevidade, à forte fidelidade local, ao crescimento lento e à formação de agregações (Beets \& Friedlander, 1992; Morris et al., 2000).

Em estudo anterior, as principais causas apontadas por pescadores da Praia Grande para a redução dos pescados foram: em primeiro lugar, arrasto, traineira ou parelha, seguidos pela grande quantidade de barcos grandes, pela pesca predatória, pelo cerco do robalo e pelo desrespeito ao defeso (Oliveira, 2010). Nota-se, portanto, que os problemas levantados se repetem. Segundo o autor, arrasto, traineira ou parelha foram as razões mais citadas em sete das treze comunidades estudadas de Paraty.

Quanto às soluções apontadas pelos pescadores, estas variaram de acordo com a espécie citada. Isso sugere que os pescadores apontam razões e soluções específicas, baseando as suas respostas em observações a respeito da dinâmica da pesca na região. Entre as sugestões para melhorar a pesca artesanal feitas por pescadores de Paraty em estudo anterior (Begossi, 2010b), destacaram-se a fiscalização correta, a proibição do arrasto, a organização dos pescadores, a proibição do cerco do robalo e o apoio do governo, além de sugestões específicas relacionadas ao defeso do camarão. Observa-se, portanto, que os estudos mostram que as principais sugestões se relacionam à fiscalização e à melhoria na regulamentação de tecnologias específicas, como arrasto e cerco com mergulho.
Esses resultados indicam que os pescadores, no que tange à capacidade de aprendizagem, mostraram-se conscientes das mudanças, em termos de abundância dos recursos pesqueiros, e do papel dos humanos nessas alterações, por meio do uso de tecnologias de pesca específicas. Os pescadores, ao apontarem soluções para o quadro de decréscimo das espécies-alvo, mostraram-se conscientes também de que, assim como a ação humana pode ser degradadora do ambiente natural, pode intervir positivamente para a mudança de cenário (Cinner et al., 2009). As soluções apontadas, em sua maioria, relacionaram-se à regulamentação de determinadas tecnologias de pesca. Isso indica, do ponto de vista da capacidade de aprendizagem, que os pescadores, ao estabelecerem as conexões entre a atividade humana e as condições em que se encontram os recursos pesqueiros, possivelmente, estarão dispostos a experimentar alternativas de gestão (Cinner et al., 2009). Begossi (2004), ao abordar exemplos bem sucedidos de comanejo no Brasil, relata que, para o processo de implantação da Reserva Extrativista do Alto do Juruá, houve demanda local do Conselho Nacional dos Seringueiros. Segundo a autora, essa demanda local para a conservação de uma área legitimou a intenção da conservação, uma vez que houve percepção local a respeito da necessidade de manutenção dos recursos naturais.

\section{Conclusões}

Foram identificados, na região estudada, os seguintes fatores que colaboram para a adaptação frente a mudanças no sistema: a flexibilidade de formas de obtenção de renda, as quais têm o potencial de reduzir a pressão sobre os recursos pesqueiros e a dependência exclusiva em relação a eles; a percepção do papel dos humanos na alteração da abundância dos recursos pesqueiros, o que indica a predisposição em 
colaborar com medidas de conservação do recurso e apontar soluções com tal finalidade.

Em contrapartida, como fatores que ameaçam a resiliência socioecológica, foram identificadas a baixa participação ou a participação não direta nas tomadas de decisão sobre a pesca, o que se relaciona, possivelmente, à frágil coesão social, à ausência de instituições locais legitimadas e aos conflitos entre pescadores e agências governamentais que regulamentam o acesso aos recursos. A partir do indicador flexibilidade, observou-se que, embora haja diversificação no uso de diferentes tecnologias de pesca e atividades, ocorrem também estratégias de intensificação, com o uso de tecnologias de maior impacto, como o arrasto.

Assim, a análise do sistema à luz da resiliência socioecológica permitiu identificar: (i) os atributos que permitem as comunidades de pescadores artesanais estudadas se adaptarem a diferentes contextos e (ii) os fatores que conferem vulnerabilidade às mesmas comunidades diante de mudanças.

Identificados tais fatores, são necessárias medidas para que o pescador possa manter seu modo de vida, se assim desejar, e para que seja assegurada a exploração sustentável dos recursos pesqueiros pela pesca artesanal. Essas medidas podem ser resumidas da seguinte maneira:

a) investimentos públicos em estratégias de diversificação, por meio do uso de diferentes tecnologias seletivas e de menor impacto e da exploração de novos nichos econômicos que valorizem o saber local, como o turismo de base comunitária;

b) incentivos do governo, na forma de financiamento para a compra de novos materiais de pesca em substituição àqueles de maior impacto;

c) promoção de confiança entre pescadores e órgãos governamentais, na forma de ins- tituições locais legitimadas. A construção de confiança demanda tempo e requer proximidade entre os atores envolvidos, com o objetivo de reunir os pontos fortes e as contribuições de cada um na regulamentação dos recursos pesqueiros.

d) investimentos públicos na educação, que contribuam, entre outras coisas, para a construção de capacidades para liderança e para estratégias que favoreçam a ação coletiva. A construção e a sustentação da coesão social são fundamentais nesse processo, pois ações individuais de participação, sem apoio ou reconhecimento da comunidade, não favorecem comportamentos de reciprocidade.

e) investimentos em infraestrutura básica são imprescindíveis para a manutenção dos benefícios fornecidos pelo ecossistema.

Os resultados apresentados somam-se a outros recentemente publicados no âmbito da pesquisa sobre a pesca na Baía da Ilha Grande, na medida em que lança o olhar especificamente sobre o componente da resiliência e sua relação com a conservação dos recursos pesqueiros e da pesca artesanal. Adicionalmente, o uso dos indicadores mostrou-se uma ferramenta útil para a caracterização dos fatores que contribuem para a construção da resiliência em escala local.

\section{Agradecimentos}

Gostaríamos de agradecer os financiamentos que tornaram este estudo possível. Agradecemos ao IDRC e FAPESP ( N ${ }^{\circ}$ 104519-004 e 2009/11154-3, respectivamente), a bolsa de mestrado FAPESP dos autores (11/15629-6 e 11/14701-5) e ainda, a participação e apoio dos pescadores da Praia Grande e Ilha do Araújo. 


\section{Referências}

Adger, W. N. Social and ecological resilience: are they related? Progress in Human Geography, 24(3), 347-364, 2000. doi: 10.1191/030913200701540465

Adger, W. N.; Brown, K.; Tompkins, E. L. The political economy of cross-scale networks in resource co-management. Ecology and Society, 10(2), 9, 2005. Disponível em: <http:// www.ecologyandsociety.org/vol10/iss2/art9/>

Anderies, J. M.; M. A. Janssen; Ostrom, E. A framework to analyze the robustness of social-ecological systems from an institutional perspective. Ecology and Society, 9(1): 18, 2004. Disponível em: $<$ http://www.ecologyandsociety.org/ vol9/iss1/art18/>

Bailey, C.; Pomeroy, C. Resource dependency and development options in coastal South East Asia. Society and Natural Resources, 9(2), 191-199, 1996. doi: 10.1080/08941929609380964

Beets, J.; Friedlander, A. Stock analysis and management strategies for red hind, Epinephelus guttatus, in the US Virgin Islands. Proceedings of the Gulf and Caribbean Fisheries Institute, 42, 66-79, 1992.

Begossi, A. Fishing spots and sea tenure: incipient forms of local management in Atlantic Forest coastal communities. Human Ecology, 23(3), 387-406, 1995. Disponível em: $<$ http://link.springer.com/article/10.1007/BF01190138>

Begossi, A. Áreas, pontos de pesca e pesqueiros na pesca artesanal. In: Begossi, A (Org.); Leme, A.; Seixas, C. S.; De Castro, F.; Pezzuti, J.; Hanazaki, N.; Peroni, N.; Silvano, R. A. M. Ecologia de pescadores da Mata Atlântica e da Amazônia. São Paulo: Hucitec, 2004. p. 187-222.

Begossi, A. The ethnoecology of Caiçara metapopulations (Atlantic Forest, Brazil): ecological concepts and questions. Journal of Ethnobiology and Ethnomedicine, 2(1), 40, 2006. doi: 10.1186/1746-4269-2-40

Begossi, A. Introdução. In: Begossi, A. (Org.); Lopes, P. F.; Oliveira, L. E. C.; Nakano, H. Ecologia de pescadores artesanais da baía da Ilha Grande. São Carlos: Rima, 2010a. p. 1-14.

Begossi, A. O manejo da pesca artesanal. In: Begossi, A (Org.); Lopes, P. F.; Oliveira, L. E. C.; Nakano, H. Ecolo- gia de pescadores artesanais da baía da Ilha Grande. São Carlos: Rima, 2010b. p. 179-234.

Begossi, A.; May, P. H.; Lopes, P. F.; Oliveira, L. E.; Da Vinha, V.; Silvano, R. A. Compensation for environmental services from artisanal fisheries in SE Brazil: Policy and technical strategies. Ecological Economics, 71, 25-32, 2011. doi:10.1016/j.ecolecon.2011.09.008

Begossi, A.; Salyvonchyk, S.; Nora, V.; Lopes, P.; Silvano, R. A. M. The Paraty Artisanal Fishery (southeastern Brazilian coast): Ethnoecology and Management of a Social-ecological System (SES). Journal of Ethnobiology and Ethnomedicine, 8, 1, 2012. doi: 10.1186/1746-4269-8-22

Begossi, A.; Nora, V.; Barreto, T.; Salyvonchyk, S.; Silvano, R. A. M. Pesca e modelos de decisão: forrageio ótimo. In: Begossi, A.; Lopes, P. F. M. (Orgs.). Comunidades pesqueiras de Paraty: sugestões para o manejo. v. 1. São Carlos: Rima, 2014. p. 59-83.

Berkes, F.; Folke, C. Linking social and ecological systems for resilience and sustainability. In: Berkes, F.; Folke, C. (Orgs.). Linking social and ecological systems: management practices and social mechanisms for building resilience. Cambridge: Cambridge University Press, 1998. p. 1-25.

Berkes, F.; Mahon, R.; McConney, P.; Pollnac, R.; Pomeroy, R.; Kalikoski, D. C. (Org.). Gestão da pesca de pequena escala: diretrizes e métodos alternativos. Rio Grande: Editora FURG, 2006.

Brasil. Decreto $n^{\circ} 7.655$ de 23.12.2011. Regulamenta a Lei $\mathrm{n}^{\mathrm{o}} 12.382$, de 25 de fevereiro de 2011, que dispõe sobre o valor do salário mínimo e a sua política de valorização de longo prazo. Brasília: DOU de 26/12/2011.

Biernacki, P.; Waldorf, D. Snowball sampling: Problems and techniques of chain referral sampling. Sociological Methods \& Research, 10(2),141-163, 1981.

Breton, Y.; Plante, S. A gestão dos recursos de uso comum em Parati: pesca e patrimônio nacional. In: Diegues, A. C. (Org.); Breton, Y.; Plante, S.; Benazera, C.; Cavanagh, J. Enciclopédia Caiçara, v. 3: o olhar estrangeiro. São Paulo: HUCITEC/NUPAUB, 2005.

Burgos, A.; Mertens, F. Os desafios do turismo no contexto da sustentabilidade: as contribuições do turismo de base co- 
munitária. Revista de Turismo y Patrimonio Cultural, 13(1), 57-71, 2015. Disponível em: <http://www.pasosonline.org/ en/articulos/download/file?fid=57.749>.

Carpenter, S.; Walker, B.; Anderies, J. M.; Abel, N. From metaphor to measurement: resilience of what to what? Ecosystems, 4(8), 765-781, 2001. doi: 10.1007/s10021001-0045-9

Cinner, J.; Fuentes, M. M. P. B.; Randriamahazo, H. Exploring social resilience in Madagascar's marine protected areas. Ecology and Society, 14(1), 41, 2009.

Davidson, J. L.; Van Putten, I. E.; Leith, P.; Nursey-Bray, M.; Madin, E. M.; Holbrook, N. J. Toward operationalizing resilience concepts in Australian marine sectors coping with climate change. Ecology and Society, 18(3), 4, 2013. Disponível em: <http://www.ecologyandsociety.org/vol14/ iss $1 / \operatorname{art} 41 />$

Diegues, A. C. Pescadores, camponeses e trabalhadores do mar. São Paulo: Ática, 1983.

Drew, J. A. Use of traditional ecological knowledge in marine conservation. Conservation Biology, 19(4), 1286-1293, 2005. doi: 10.1111/j.1523-1739.2005.00158.x

Filgueiras, M. D. P. Tempo e espaço entre pescadores da Praia da Concha, Vila Velha-Es. Sociedade em Estudos, 2(2), 30-38, 2007. Disponível em: <http://www.sociedadeemestudos.ufpr.br/atual/arquivos/filgueiras\%20pag\%2030.pdf>

Gunderson, L. Resilience, flexibility and adaptive management - antidotes for spurious certitude. Conservation Ecology, 3(1), 7, 1999. Disponível em: <http://dlc.dlib. indiana.edu/dlc/handle/10535/2472>

Hanazaki, N.; Berkes, F.; Seixas, C. S.; Peroni, N. Livelihood diversity, food security and resilience among the Caiçara of Coastal Brazil. Human Ecology, 41(1), 153164, 2013. Disponível em: <http://link.springer.com/article/10.1007/s10745-012-9553-9>

Holling, C. S. Understanding the complexity of economic, ecological, and social systems. Ecosystems, 4(5), 390405, 2001. Disponível em: <http://link.springer.com/article/10.1007/s10021-001-0101-5>

Hughes, T. P.; Bellwood, D. R.; Folke, C.; Steneck, R. S.; Wilson, J. New paradigms for supporting the resilience of marine ecosystems. Trends in ecology \& evolution, 20(7), 380-386, 2005. doi:10.1016/j.tree.2005.03.022
Jennings, S.; Revill, A. S. The role of gear technologists in supporting an ecosystem approach to fisheries. Journal of Marine Science: Journal du Conseil, 64(8), 1525-1534, 2007. doi: 10.1093/icesjms/fsm104

Jentoft, S. The community: a missing link of fisheries management. Marine Policy, 24(1), 53-60, 2000. doi: 10.1016/ S0308-597X(99)00009-3

Johannes, R. E.; Freeman, M. M.; Hamilton, R. J. Ignore fishers' knowledge and miss the boat. Fish and Fisheries, 1(3), 257-271, 2000. doi: 10.1111/j.1467-2979.2000.00019.x

Joventino, F. K. P.; Lianza, S.; Johnsson, R. M. F. Pesca artesanal na Baía de Ilha Grande, no Rio de Janeiro: conflitos com unidades de conservação e novas possibilidades de gestão. Política \& Sociedade, 12(23), 159-182, 2013.

Lopes, P. F. O pescador artesanal da Baía da Ilha Grande. In: Begossi, A. (Org.); Lopes, P. F.; Oliveira, L. E. C.; Nakano, H. Ecologia de pescadores artesanais da baía da Ilha Grande. São Carlos: Rima, 2010a. p. 15-72.

Lopes, P. F. A pesca na Baía da Ilha Grande: caracterização e conflitos. In: Begossi, A. (Org.); Lopes, P. F.; Oliveira, L. E. C.; Nakano, H. Ecologia de pescadores artesanais da baía da Ilha Grande. São Carlos: Rima, 2010b. p. 101-178.

Lopes, P. F.; Silvano, R. A.; Begossi, A. Extractive and Sustainable Development Reserves in Brazil: resilient alternatives to fisheries? Journal of Environmental Planning and Management, 54(4), 421-443, 2011. doi: 10.1080/09640568.2010.508687

Lopes, P. F. M.; Rosa, E. M.; Salyvonchyk, S.; Nora, V.; Begossi, A. Suggestions for fixing top-down coastal fisheries management through participatory approaches. Marine Policy, 40, 100-110, 2013. doi:10.1016/j.marpol.2012.12.033

Maldonato, C. O turismo rural comunitário na América Latina: gênesis, características e políticas. In: Bartholo, R.; Sansolo, D. G.; Bursztyn, I. (Orgs.). Turismo de base comunitária: Diversidade de olhares e experiências brasileiras. Rio de Janeiro: Letra e Imagem, 2009.

Marshall, N. A.; Fenton, D. M.; Marshall, P. A.; Sutton, S. G. How Resource Dependency Can Influence Social Resilience within a Primary Resource Industry. Rural Sociology, 72(3), 359-390, 2007. doi: 10.1526/003601107781799254

McCay, B. J. Systems ecology, people ecology, and the anthropology of fishing communities. Human Ecology, 6(4), 397-422, 1978. doi: 10.1007/BF00889417 
Morris, A. V.; Roberts, C. M.; Hawkins, J. P. The threatened status of groupers (Epinephelinae). Biodiversity \& Conservation, 9(7), 919-942, 2000. doi: 10.1023/A:1008996002822

Nora, V. Ecologia e Etnoecologia de Robalos (Centropomus undecimalis, Bloch, 1792 e Centropomus parallelus, Poey, 1860) na Baía de Paraty, RJ, Brasil. Santos, Dissertação (Mestrado em Ecologia) - Universidade Santa Cecília, 2013. Disponível em: < http://sites.unisanta.br/ppgecomar/ dissertacoes.asp $>$

Oliveira, L. E. C. A percepção da conservação na Baía da Ilha Grande. In: Begossi, A. (Org.); Lopes, P. F.; Oliveira, L. E. C.; Nakano, H. Ecologia de pescadores artesanais da baía da Ilha Grande. São Carlos: Rima, 2010. p. 235-286.

Ostrom, E. Collective action and the evolution of social norms. The Journal of Economic Perspectives, 14(3), 137158, 2000. doi: 10.1080/19390459.2014.935173

Ostrom, E. A General Framework for Analyzing Sustainability of Social-Ecological Systems. Science, 325, 419, 2009. doi: 10.1126/science. 1172133

Prado, D. S.; Seixas, C. S.; Berkes, F. Looking back and looking forward: Exploring livelihood change and resilience building in a Brazilian coastal community. Ocean \& Coastal Management, 113, 29-37, 2015. doi: 10.1016/j. ocecoaman.2015.05.018
Seixas, C.; Berkes, F. Mudanças socioeológicas na pesca da Lagoa de Ibiraquera, Brasil. In: Vieira, P. F.; Berkes, F.; Seixas, C (Orgs.). Gestão integrada e participativa de recursos naturais. Florianópolis: SECCO/APED, 2005.

Silvano, R. A.; MacCord, P. F.; Lima, R. V.; Begossi, A. When does this fish spawn? Fishermen's local knowledge of migration and reproduction of Brazilian coastal fishes. Environmental Biology of Fishes, 76(2-4), 371-386, 2006. doi: 10.1007/s10641-006-9043-2

Trimble, M. Towards Adaptive Co-management of Artisanal Fisheries in Coastal Uruguay: Analysis of Barriers and Opportunities, with Comparisons to Paraty (Brazil). Winnipeg, Thesis (Phd in Philosophy) - Natural Resources Institute, University of Manitoba, 2013.

Trimble, M.; Johnson, D. Artisanal fishing as an undesirable way of life? The implications for governance of fishers' wellbeing aspirations in coastal Uruguay and southeastern Brazil. Marine Policy, 37, 37-44, 2013. doi:10.1016/j. marpol.2012.04.002

Zar, J. H. Biostatistical analysis. $4^{\text {th }}$ ed. Upper Saddle River, NJ: Prentice Hall, 1999. p. 389-394.

Walker, B.; Carpenter, S.; Anderies, J.; Abel, N.; Cumming, G.; Janssen, M.; Pritchard, R. Resilience management in social-ecological systems: a working hypothesis for a participatory approach. Conservation Ecology, 6(1), 14, 2002. 